\title{
OBITUARY
}

\section{Setsuro Ebashi (1922-2006)}

\author{
Physiologist who uncovered the regulatory role of calcium in cells.
}

All animal movement depends on muscle contractions. Nerve impulses cause a change of electrical potential across the muscle-cell membrane, which rapidly propagates to both ends of the cell, causing contraction. How electrical excitation at the muscle cell's surface induces contraction of the proteins packed inside was one of the biggest mysteries in physiology for decades. It was Setsuro Ebashi, who died on 17 July 2006 at the age of 83 , who essentially solved this conundrum.

Muscle cells contain two types of filament that are aligned along the long axis of the cell. Contraction occurs when the protein myosin in the 'thick' filaments reacts with another protein, actin, in the 'thin' filaments, causing the filaments to slide across each other, pulling the ends of the cell closer together. The process is fuelled by the breakdown of ATP. The biochemical basis of contraction was largely determined by Albert Szent-Györgyi in the 1940s, and the biophysical and structural basis was uncovered by Jean Hanson and Hugh Huxley, and by Andrew Huxley and R. Niedergerke, during the 1950s. But how the process was triggered by nerves remained a mystery.

Ebashi discovered that in the absence of calcium ions, no contractile reaction occurs, even when ATP is added to the myosin-actin system, but that with even a minute amount of calcium (of the order of 1 micromolar), ATP induces a vigorous contractile reaction. This calcium-dependence had been previously overlooked by biochemists because of low-level contamination from laboratory glassware and impurities in the chemical reagents. Ebashi took tremendous pains to avoid contamination by calcium ions in all the solutions and protein preparations he used, making his results unequivocal. His work was consistent with contemporary results from Annemarie Weber, who showed independently that the breakdown of ATP during the contractile reaction requires minute amounts of calcium.

The idea that calcium might be involved in muscle contraction came to Ebashi during his studies on the 'relaxing factor' reported by B. B. Marsh in 1951. This was a fraction of the homogenate that is made when muscle cells are ground up, and it could induce relaxation of the myosin-actin system. Ebashi proved that relaxing factor is nothing but fragmented pieces of a muscle-specific organelle called the sarcoplasmic reticulum. Enquiring into the factor's mechanism of action, Ebashi got a hint from Emil Bozler's result in 1954 that EDTA - a 'chelating' agent that sequesters calcium - causes relaxation. Ebashi compared the calcium-binding activity of various chelating agents with their relaxing activity and found that they correlated exactly. He further showed that fragmented sarcoplasmic reticulum can accumulate calcium ions rapidly in the presence of ATP, and so can remove enough calcium from the surrounding medium to cause relaxation.

The relaxation process is the reverse of contraction, so Ebashi proposed what is our current understanding of excitationcontraction coupling: excitation at the surface membrane somehow sends a signal to the sarcoplasmic reticulum; this releases the calcium ions that accumulate there during the relaxing and resting period, and the flood of calcium ions induces the contractile reaction.

Examining the process more closely, Ebashi discovered that purified myosin and actin react with ATP even in the complete absence of calcium ions, and that the regulatory action of calcium is exerted only in the presence of a certain protein factor. This factor turned out to be a mixture of tropomyosin, a protein that previously had no known function, and a newly discovered protein that Ebashi named troponin. Tropomyosin and troponin are present with actin in the thin filaments. In the absence of calcium ions, the two proteins cooperate to inhibit actin, preventing it from interacting with myosin in the thick filaments. Having found that calcium binds strongly to troponin, Ebashi proposed that the resulting conformational changes in troponin are transmitted through tropomyosin to actin to remove the inhibition, and the contractile reaction ensues - a mechanism that has subsequently been confirmed.

In the early 1960s, the idea that a simple inorganic ion such as calcium controls contraction was not popular among most biochemists - the prevailing belief was that such an important biological phenomenon as contraction should be regulated by sophisticated organic molecules. So Ebashi had a hard time getting his ideas taken seriously, despite his clear evidence. Only after the discovery of troponin and his elucidation of the mechanism did everybody accept the regulatory role of calcium ions.

The regulatory roles of calcium are not confined to muscle contraction. Since Ebashi's discovery, numerous cellular processes, including the release of neurotransmitters and hormones, metabolic switching and gene expression, have been found to be controlled by calcium. Thus,

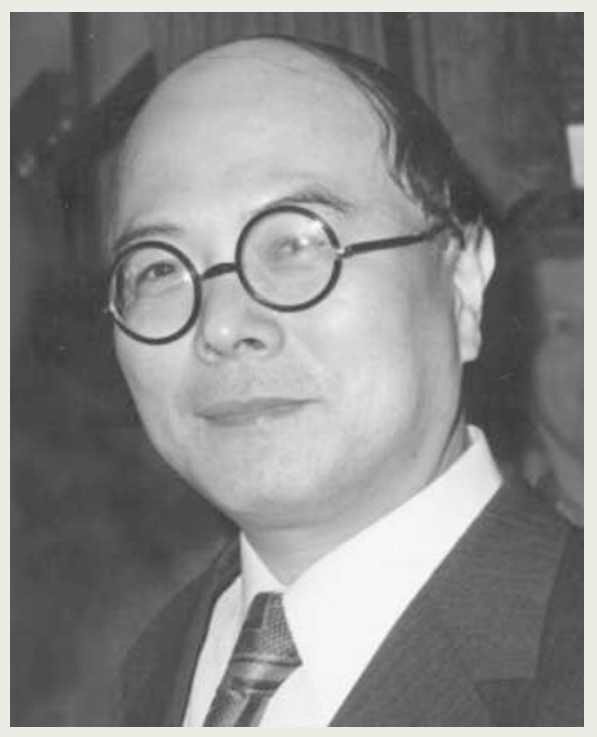

Ebashi really broke open the field of calcium signalling, and profoundly influenced life science as a whole.

Setsuro Ebashi was brilliant from childhood. He skipped a year in both primary and middle schools and was admitted into the Dai-ichi High School, the most prestigious high school in Japan at the time. He was promoted to be professor of pharmacology at the University of Tokyo at the age of 36. But his scientific success was not only due to his sharp mind - he worked extremely hard in the laboratory, until after midnight most nights. Although he had many collaborators, most notably his wife Fumiko Ebashi, all the major results were, amazingly, produced by Ebashi himself.

As one of the most respected scientists in physiology and pharmacology worldwide, Ebashi received numerous honours, including the Order of Cultural Merit, the highest scientific honour in Japan. He was a Member of the Japan Academy and a Foreign Member of the Royal Society in London, and of several other academies, including those of the United States, Germany and Belgium. $\mathrm{He}$ also contributed to the global scientific community as president of the International Union for Pure and Applied Biophysics (1978-81) and the International Union of Pharmacology (1990-94), and presided over the International Congress of Pharmacology held in Tokyo in 1981.

Ebashi was a man of attractive personality: charismatic, warm-hearted, helpful and loyal to his colleagues, and patriotic to his country. Despite suffering physical handicaps following a stroke in 2000, he kept a clear mind, and because his condition was fairly stable, nobody expected his sudden death. All his friends and pupils greatly lament it.

\section{Makoto Endo}

Makoto Endo is in the Department of

Pharmacology, University of Tokyo, 7-3-1,

Bunkyo-ku, Tokyo 113-0033, Japan.

e-mail:makoendo@gakushikai.jp 